\title{
Aspectos Biológicos da L-Glutamina: Iimunomodulação e Hipertrofia Muscular - Estudo ao Longo do Tempo
}

\author{
Biological Aspects of L-Glutamine: Immunomodulation and \\ Muscle Hypertrophy - Follow-Up Study
}

Mateus Dantas Moraes Freire ${ }^{1}$, Natalia Maria Bezerra Lucas das Chagas ${ }^{2}$, Samanta Queiroz dos Santos ${ }^{3}$, Songelí Menezes Freire ${ }^{4}$, André Ney Menezes Freire ${ }^{5^{*}}$ ${ }^{1}$ Acadêmico de Medicina (FTC); ${ }^{2}$ Acadêmica de Nutrição (UFBA); ${ }^{3}$ Professora de Análises Clínicas, Ouvidora da Policlínica Regional Metrô Recôncavo Norte/BA; ${ }^{4}$ Professora Associada de Bioética e de Biossegurança do Instituto de Ciências da Saúde. da Universidade Federal da Bahia (UFBA); ${ }^{5}$ Professor Titular de Cirurgia da UFBA. Coordenador do Serviço de Nutrição Enteral e Parenteral-SENEP-HSI; Salvador, Bahia, Brasil

Correspondence addresses: Dr. André Ney Menezes Freire andreney.freire@gmail.com

Received: December 18, 2019

Revised: January 16, 2020

Accepted: Janruary 22, 2020

Published: March 31, 2020

Data Availability Statement: All relevant data are within the paper and its Supporting Information files.

Funding: This work was the result of authors' initiative. There was no support of research or publication funds.

Competing interests: The authors have declared that no competing interests exist.

Copyright

(C) 2020 by Santa Casa de Misericórdia da Bahia. All rights reserved.

ISSN: 2526-5563
O uso do aminoácido L-glutamina (GLN), entre outros aminoácidos e biomoléculas, tem sido extensivo nas abordagens terapêuticas nos últimos anos. $O$ objetivo desse estudo foi o de trazer à luz os principais achados da literatura nacional e internacional sobre o uso da glutamina, além de fundamentar a importância básica e clínica para a leitura crítica da comunidade sobre o tema. Foi realizada releitura de 79 artigos publicados de 1988 a 2020. Os artigos com abordagem experimental in vitro ou in vivo em humano foram categorizados segundo origem dos autores, origem e amostragem da população de estudo, tipo de demanda/ indicação clínica, resultado favorável ou desfavorável da suplementação com GLN. Entre os anos de 1995 a 2020 foram encontrados 9 trabalhos experimentais in vivo no modelo humano com suplementação com GLN com significativo efeito positivo na maioria. As abordagens foram com população de estudo alvo infantil com bebês, adolescentes, homens e mulheres. Foram encontrados estudos com foco na performance física, na minimização da infecção, na cirurgia, oncologia e diminuição de morbidades diversas. Os dois artigos experimentais in vitro com amostras humanas, analisados, referiam à importância da glutamina em mecanismos fisiológicos de defesa, de funcionalidade e de integridade celular. Entre os trabalhos analisados, disponíveis sobre esses temas, o uso da suplementação com a GLN foi descrita mais como favorável e de sucesso para as demandas estabelecidas pela equipe médica, com variáveis doses e intervalos de uso. Entre os artigos identificados com trabalhos nessas abordagens, com doses e tempos de uso nas diferentes morbidades ou demandas, a variação foi encontrada em baixas doses, dentro do recomendado internacionalmente. Não foram encontrados artigos com ênfase em aspectos negativos sobre o uso da glutamina, embora devase considerar o cuidado e analisar, caso a caso, com acompanhamento clínico constante pela equipe médica que estabeleça o seu uso.

Palavras-chave: Glutamina; GLN; Suplementação; Imunonutrição; Hipertrofia Muscular.

The use of the amino acid L-glutamine (GLN), among other amino acids and biomolecules, has been extensive in therapeutic approaches in recent years. This study 
aimed to highlight the findings of national and international literature regarding the use of glutamine. Seventy-nine articles published from 1988 to 2020 were studied. Articles with an experimental in vitro or in vivo approach to humans were categorized according to the origin of the authors, origin, and sampling of the study population, type of demand / clinical indication, a favorable or unfavorable result of GLN supplementation. Between 1995 and 2020, 9 in vivo experimental studies were found in the human model with GLN supplementation, with a significant positive effect in almost them. The approaches were with a target child study population with babies, adolescents, men, and women. Studies were found focusing on physical performance, minimizing infection, surgery, oncology, and reduction of morbidities. The two experimental articles in vitro with human samples, analyzed, referred to the importance of glutamine in physiological mechanisms of defense, functionality, and cell integrity. Among the studies analyzed, available on these topics, the use of supplementation with GLN was described as more favorable and successful for the demands established by the medical team, with varying doses and intervals of use. Among the articles identified with these approaches, with doses and times of use in different morbidities or demands, the variation was found in low doses, within the internationally recommended. No articles were found with an emphasis on negative aspects of the use of glutamine, although care should be considered and analyzed, case by case, with constant clinical monitoring by the medical team that establishes its use. Keywords: Glutamine; GLN; Supplementation; Immunonutrition; Muscular Hypertrophy.

\section{Introdução}

Entre as possibilidades de suplementação nutricional no suporte a demandas metabólicas encontram-se a nutrição parenteral e enteral com possibilidade de diversos nutrientes, entre eles a glutamina.

\section{Glutamina (GLN)}

A glutamina (GLN) é um aminoácido com massa molecular de $146,14 \mathrm{~g} / \mathrm{mol}$ que o organismo consegue sintetizar/produzir em níveis adequados às necessidades biológicas em condições de equilíbrio metabólico, sendo, portanto, considerado um aminoácido não essencial para indivíduos saudáveis e em condições normais. Por outro lado, mesmo sendo o aminoácido mais abundante no fluido intra e extracelular, em situações especiais como de traumas graves, infecções, desnutrição, doenças do sistema imunológico e do sistema digestório, inflamações graves, cânceres, atividades físicas intensas ou extenuantes, a GLN se torna condicionalmente essencial.

A glutamina foi identificada em 1883 pelos pesquisadores Ernst Schulze, químico alemão e por Emil Bosshard, químico suíço, e isolada do suco da beterraba, e mostrou-se que este importante aminoácido está presente tanto no reino vegetal quanto no reino animal

\section{Características Bioquímicas da Glutamina}

A glutamina possui uma limitada solubilidade em água: $35 \mathrm{~g} / \mathrm{L}$ de água, a $20^{\circ} \mathrm{C}$, podendo um grama ser dissolvido em $20,8 \mathrm{~mL}$ de água a $30^{\circ} \mathrm{C}$, em $38,5 \mathrm{~mL}$ a $18^{\circ} \mathrm{C}$, em $56,7 \mathrm{~mL}$ a $0^{\circ} \mathrm{C} .1,2$ Entretanto, o uso do dipeptídeo L-alanil-Lglutamina (peso molecular de 217,22 g/mol) é um composto estável usado em soluções de nutrição parenteral e possui solubilidade de $586 \mathrm{~g} / \mathrm{L} .{ }^{1}$

A glutamina participa de diferentes funções no corpo e dentre elas está a proliferação e desenvolvimento de células, obalançoácido-base, o transporte da amônia entre os tecidos, a doação de esqueletos de carbono para a gliconeogênese, a participação no sistema antioxidante, dentre outras funções. ${ }^{3,4}$ É bioquimicamente importante como constituinte das proteínas, além de ser essencial para o metabolismo do nitrogênio. Atua como doador de amônia e posterior formação de ureia e purinas, sendo a primeira excretada pelos rins e a segunda utilizada na formação de ácidos nucleicos.

\section{Principais Alimentos que Contém Alta Concentração de Glutamina/Ácido Glutâmico em sua Composição}

Foram resgatadas algumas informações alimentares, com dados da Tabela Brasileira de Composição de Alimentos $(\mathrm{TACO})^{5}$ e 
complementados com dados da planilha do Programa de Nutrição da Escola Paulista de Medicina, disponível on line (Tabela 1). ${ }^{6}$ Segundo observado em uma lista de categorização de riqueza de composição dos alimentos, cada $100 \mathrm{~g}$ de beterraba crua consta $0.45 \mathrm{~g}$ de glutamato e da folha de beterraba cozida, $0.31 \mathrm{~g}$, sendo o glutamato, entre os 2.594 alimentos, um dos mais representativos de origem animal e vegetal.

\section{Síntese, Captação e Distribuição de Glutamina no Corpo Humano}

Krebs, em 1935, demonstrou a capacidade das células de sintetizar e degradar a glutamina no organismo humano. A glutamina é sintetizada no nosso corpo a partir do glutamato em praticamente todos os tecidos e órgãos, como músculo esquelético, pulmão, cérebro, fígado e, possivelmente, o tecido adiposo, que apresentam elevada atividade da enzima glutamina sintetase, sendo assim considerados tecidos sintetizadores de glutamina. ${ }^{7,8}$

A síntese da glutamina é feita a partir do ácido glutâmico (a glutamina é a amida do ácido glutâmico), valina e isoleucina. A glutamina sintetase catalisa a síntese de glutamina fazendo a interação de glutamato e amônia, e a glutaminase faz a reação inversa. A taxa de síntese deste aminoácido no músculo esquelético humano é de aproximadamente $50 \mathrm{mmol} / \mathrm{h}$, sendo maior do que qualquer outro aminoácido. ${ }^{8}$ A glutamina pode ser também sintetizada a partir da captação de alimentos e da transformação a partir de outros aminoácidos.

A distribuição de glutamina no corpo humano é responsável pelos seus efeitos benéficos no organismo, constitui $25 \%$ dos aminoácidos livres no plasma e $60 \%$ dos aminoácidos do músculo esquelético.

O principal tecido de síntese, estoque e liberação de glutamina é o tecido muscular esquelético. ${ }^{9}$ Os músculos precisam ajustar o consumo de aminoácidos com a exportação da amônia para o fígado na forma dos aminoácidos glutamina ou alanina, em uma via metabólica extremamente importante e que permite o equilíbrio fisiológico, principalmente durante a realização de exercícios físicos.

No sangue, a concentração de glutamina é em torno de $500-700 \mu \mathrm{mol} / \mathrm{L}^{10}$ sendo que indivíduos pesando aproximadamente $70 \mathrm{~kg}$ apresentam cerca de 70-80g de glutamina, distribuída por diversos tecidos corporais. Tanto a concentração tecidual quanto a concentração sanguínea de glutamina podem ser influenciadas pela atividade da glutamina sintetase ou da glutaminase. Alguns tipos de células, tais como células do sistema imune, rins e intestino, apresentam elevada atividade de glutaminase, sendo assim considerados tecidos consumidores de glutamina. ${ }^{9}$

\section{Funções da Glutamina no Corpo}

No cérebro, este aminoácido é utilizado como um importante agente na redução da concentração de amônia, com consequente desintoxicação e síntese de glutamina para nova síntese de glutamato. ${ }^{11}$

Nos rins, a glutamina sintetase é imprescindível para o controle do metabolismo do nitrogênio e manutenção do $\mathrm{pH}$ no organismo. ${ }^{12}$

No pulmão e no músculo esquelético, é responsável pela manutenção da concentração de glutamina plasmática, sendo essencial em situações patológicas ou de estresse. ${ }^{13} \mathrm{O}$ transporte de amônia é realizado sob a forma de glutamina. O excesso de amônia nos tecidos é adicionado ao glutamato para formar glutamina, um processo catalisado pela glutamina sintetase. Após o transporte na corrente sanguínea, a glutamina penetra no fígado e o $\mathrm{NH}^{+}$é liberado na mitocôndria pela enzima glutaminase.

A glutamina regula positivamente as vias de sinalização celular e molecular atuando na indução da expressão de genes para produção de hormônios, citocinas e proteínas musculares, participa do transporte de nitrogênio entre os tecidos e influência no potencial redox celular por 
Tabela 1. Proporção de glutamina em alimentos de origem animal e vegetal.

\begin{tabular}{|c|c|c|c|}
\hline \multicolumn{2}{|c|}{ Alimentos de origem animal } & \multicolumn{2}{|c|}{ Alimentos de origem vegetal } \\
\hline $\begin{array}{l}\text { Alimentos } \\
\text { (100 grs) }\end{array}$ & $\begin{array}{c}\text { Glutamina } \\
\text { Glutamato (g) }\end{array}$ & $\begin{array}{l}\text { Alimentos } \\
\text { (100 grs) }\end{array}$ & $\begin{array}{c}\text { Glutamina } \\
\text { Glutamato }(\mathrm{g})\end{array}$ \\
\hline Queijo mozzarella & 4,45 & Tofu frito & 2,97 \\
\hline Carne bovina - figado & 3,33 & Milho amarelo & 1,768 \\
\hline Salmão, Rosa, Cru & 2,903 & Grão de bico, cozido & 1,55 \\
\hline Ovo, galinha, cozido & 1,644 & Lentilha, cozida & 1,399 \\
\hline Leite integral $3.5 \%$ de gordura & 0,708 & Feijão preto, cozido & 1,351 \\
\hline Iogurte natural integral & 0,679 & Feijão branco, cozido & 1,259 \\
\hline & & Ervilha verde, cozida & 0,733 \\
\hline & & Arroz branco, cozido & 0,524 \\
\hline & & Leite de soja & 0,487 \\
\hline & & Beterraba, crua & 0,428 \\
\hline & & Espinafre, cozido & 0,357 \\
\hline & & Repolho, cru & 0,27 \\
\hline
\end{tabular}

Fonte: dados extraídos da Escola Paulista de Medicina (disponível em https://tabnut.dis.epm.br/alimento - acesso em 27 de fevereiro de 2020).

induzir a produção de glutationa, um importante antioxidante intracelular. ${ }^{14}$

A glutamina participa de diversas funções celulares, sendo substrato energético para síntese de glicose, ácidos nucléicos como purinas e pirimidinas, importantes para ativação de células de proliferação rápida como linfócitos e macrófagos, ${ }^{3}$ modulando assim a resposta imune e a resposta inflamatória.

Por ser constituído por células de rápida divisão, o epitélio intestinal utiliza a glutamina como importante fonte energética e de nitrogênio amídico para a síntese de nucleotídeos. ${ }^{15,16}$ Este aminoácido também possui efeitos tróficos sobre enterócitos e, como tal, auxilia na manutenção da integridade intestinal em situações de estresse.

Há diversas funções da glutamina nos diferentes órgãos e tecidos (Figura 1). Nosúltimos anos têm sido descritos fenômenos e atividades fisiológicas no cérebro/SNC, propiciando o tamponamento da amônia; no rim, como substrato energético e excreção amônia; no intestino, como substrato energético, hipertrofia celular, absorção de eletrólitos; no fígado, também como substrato energético, síntese do glutation, metabolismo da amônia; no músculo esquelético, como substrato energético, síntese do glicogênio e glutationa, impedimento do acúmulo de amônia e atenuação do dano muscular. Nos diversos outros tecidos, foram descritas também funções como substrato energético, anti-inflamatório, citoprotetor, papel imunomodulador, anti-oxidante indireto e responsável ainda pelo aumento da síntese de glutationa. Entre as diferentes capacidades da glutamina está presente em outras funções, como proliferação celular e manutenção do equilíbrio ácido-base. Esse aminoácido começou a ser investigado na nutrição do esporte e, além de seu efeito no sistema imunológico, que atribuiu à glutamina várias propriedades, apresentou um papel importante na anti-fadiga frente ao exercício físico intenso ${ }^{17}$ e no auxílio da hipertrofia muscular.

\section{A Importância da Glutamina nas Demandas Metabólicas e em Algumas Patologias}

Nos últimos anos, muitas referências foram encontradas explicando a glutamina nas diferentes situações metabólicas e demandas 
Figura 1. Alguns dos mecanismos de modulação das atividades celulares, dos tecidos e dos órgãos pela glutamina.

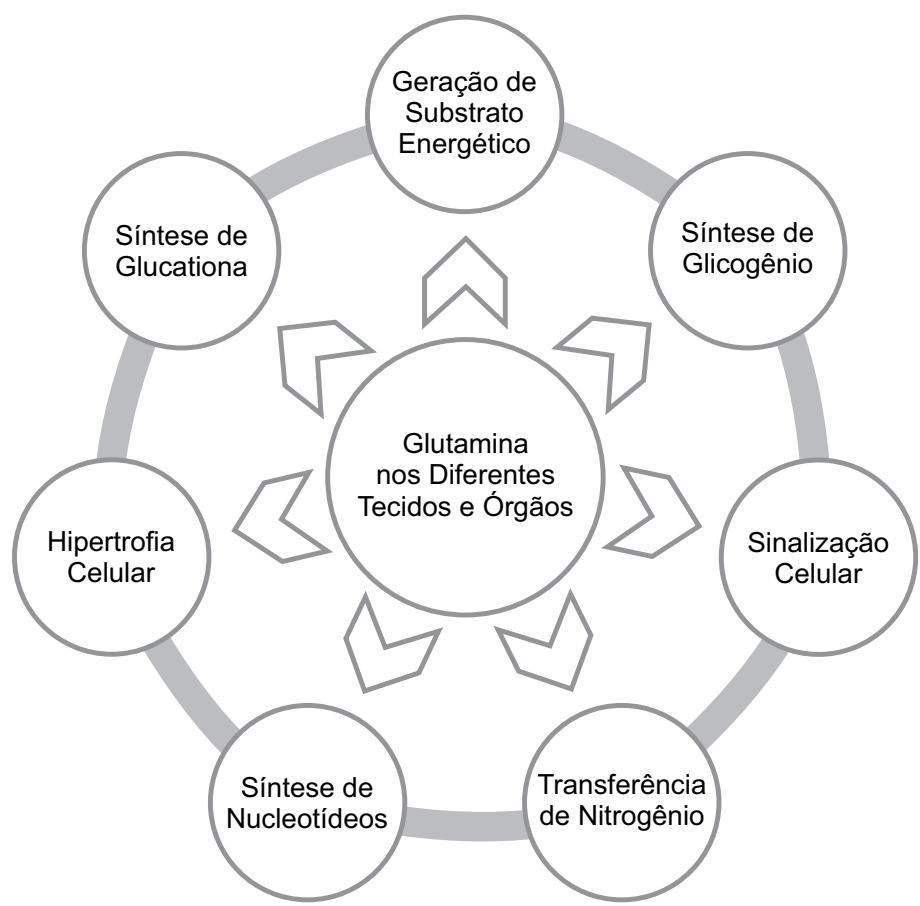

Fonte:Adaptado a partir de Borges et al. $(2008)^{4}$ e Coqueiro, Rogero e Tirapegui (2019) ${ }^{17}$.

especiais em exercícios intensos e em processos de catabolismo exacerbado, como em alguns modelos patológicos.

\section{Sistema Gastrointestinal}

Na mucosa intestinal humana, foi visto que a glutamina reduz a produção de a citocinas pró-inflamatórias como IL-6 e IL-8 e aumenta a produção de IL-10, anti-inflamatória. ${ }^{18}$ Além disso, foi sugerido que a suplementação oral com glutamina pode melhorar a resposta do sistema imune intestinal que são mediadas pelos linfócitos intraepiteliais. ${ }^{19}$

A glutamina adicionada à suplementação clássica nutricional de aminoácidos pode contribuir para a preservação do intestino, diminuindo a atrofia das vilosidades e mantendo a função intestinal, podendo constituir um fator essencial no bem-estar e na boa saúde de indivíduos muito idosos. ${ }^{20}$

Os intestinos delgado e grosso são capazes de metabolizar grandes quantidades de glutamina que são fornecidas através da dieta, pela corrente sanguínea ou por ambos. Para o intestino, a glutamina, quantitativamente, possui maior relevância como substrato energético quando comparada à glicose. ${ }^{21}$

Também se considera a hipótese de a glutamina atenuar as reações que impulsionam inflamação sistêmica mediada pelo intestino, minimizando a produção de citocinas pró-inflamatórias e aumentando as concentrações de imunoglobulina, como a IgA,${ }^{18}$ importante na defesa das mucosas, além de diminuir a permeabilidade intestinal em pacientes críticos. ${ }^{22,23}$

\section{Câncer}

A progressão do tumor está associada a um alto consumo de glutamina do hospedeiro pelas células tumorais e a uma depressão na atividade das células natural killer (NK) devido a uma diminuição nas concentrações de glutationa. 
A suplementação dietética de glutamina pode, portanto, ter o efeito benéfico de restaurar os níveis de glutationa dentro das células natural killer; ao mesmo tempo, no entanto, poderia ter o efeito deletério de alimentar o tumor. Contudo, como o consumo de glutamina pelos tumores é quase absolutamente dissipativo, não se deve esperar um aumento na taxa de crescimento do tumor devido a esse processo. De fato, existem dados experimentais que parecem indicar que um suplemento dietético diminui o crescimento do tumor ao restaurar a função das células assassinas naturais e melhora o metabolismo das proteínas do hospedeiro ou paciente. ${ }^{24}$

Segundo Medina (2001), ${ }^{24}$ existe um fluxo de glutamina do hospedeiro para o tumor e essa demanda proporciona um aumento das enzimas glutamina sintetase e glutaminase. O consumo de glutamina pelo tumor dependente da disponibilidade de outros substratos energéticos disponíveis como glicose e ácidos graxos livres. A progressão tumoral está associada à diminuição da atividade das células NK devido à diminuição da atividade da enzima glutationa nessas células. A suplementação com glutamina está relacionada com o aumento dos níveis celulares dessa enzima, conferindo melhor resposta das células NK, diminuindo crescimento tumoral e melhorando o metabolismo proteico do hospedeiro.

A utilização da glutamina no paciente oncológico está relacionada à melhora da função imunológica, muscular e intestinal, e, consequentemente, da redução de complicações infecciosas, além da melhora na tolerância à terapia antineoplásica, como foi reforçada desde a última década por Waitzberg. ${ }^{25}$

\section{Infecções, Traumas e Cirurgias}

Aprincipal fonteenergéticaparadiferenciação e proliferação dos enterócitos é a glutamina. Ela tem papel central na prevenção da translocação bacteriana, que pode levar à possibilidade de evolução para sepse e disfunção múltipla de órgão. Isso pode ser explicado pelo efeito que essa molécula tem em estimular o trofismo de células do intestino delgado e cólon. ${ }^{26}$

Em 1998, a revista The Lancet (Vol 352, November 7, 1998) publicou a resposta dos autores referente ao questionamento do artigo Glutamine-enriched enteral nutrition in patients with multiple trauma, publicado por Alexander Houdijk e colegas (na publicação de Sept 5 p 772), ${ }^{27}$ no qual a suplementação enteral com glutamina em pacientes com trauma múltiplo mostrou uma redução na incidência de pneumonia, sepse e bacteremia mas que naquele momento os resultados eram difíceis de interpretar.

Em uma coorte com 500 indivíduos, foi estudado o efeito da glutamina na resposta do hospedeiro contra o Mycobacterium tuberculosis e foi observado que esse aminoácido implica positivamente nesse processo através de inibidores específicos da via da glutamina que levam a respostas diminuídas de citocinas, principalmente interferon $\gamma$, interleucina $17 \mathrm{e}$ interleucina. ${ }^{22,28}$

\section{Atividade Física e Desempenho Físico e Muscular}

Entre os artigos encontrados, observou-se o importante papel da glutamina no desempenho físico e muscular na atividade física. $\mathrm{Na}$ revisão sobre esse tema, Rogero e Tirapegui ${ }^{29}$ já relatam importantes variáveis de estudo como intensidade e duração do exercício, estado nutricional dos indivíduos e diferenças no tempo de coleta de sangue, forma de estocagem de amostras de plasma e técnica bioquímica de medida da concentração de glutamina, que ocasionam dados equivocados e contraditórios. A subsequente redução da concentração plasmática referida, quando o exercício é realizado por mais de 1 hora, indica a necessidade da suplementação da glutamina para evitar dano inflamatório e desgaste, ou destruição do tecido muscular envolvido. 


\section{Anemia Falciforme}

Em 7 de julho de 2017, a Administração de Alimentos e Medicamentos dos EUA (FDA) ${ }^{30}$ aprovou a glutamina para o tratamento da anemia falciforme para adultos e crianças acima de 5 anos. E, mais recentemente, em 2019, a ANVISA iniciou o processo de recomendação de seu uso no Brasil.

$\mathrm{Na}$ anemia falciforme, existe a hipótese de que a glutamina sirva como substrato para a síntese de NAD, que se apresenta com capacidade de oxirredução diminuída nas células afetadas, reduzindo a importante função que o fenômeno oxidativo tem na fisiopatologia da anemia falciforme. A anemia falciforme é uma doença muito prevalente na população afrodescendente, que compõe uma grande parte da população brasileira.

\section{Doses de Suplementação}

Uma dose maior que $0,2 \mathrm{~g} / \mathrm{kg} / \mathrm{dia}$ diminuiu complicações infecciosas em pacientes cirúrgicos quando comparados com doses menores que $0,2 \mathrm{~g} / \mathrm{kg} / \mathrm{dia}$. A Sociedade Americana de Nutrição Parenteral e Enteral (ASPEN - American Society for Parenteral and Enteral Nutrition) sugere que a dose ideal de suplementação de glutamina seja próxima de $0,5 \mathrm{~g} / \mathrm{kg} / \mathrm{dia} .{ }^{31}$

Pacientes queimados ou vítimas de trauma devem ter associados ao regime de suporte nutricional enteral, 0,3 a $0,5 \mathrm{~g} / \mathrm{kg} /$ dia de glutamina suplementar divididos em 2 ou 3 doses conforme sugerido pela Sociedade Brasileira de Nutrição Parenteral e Enteral (SBNPE). ${ }^{32}$

A recomendação da ESPEN de 201933 traz informações quanto ao uso da glutamina enteral e parenteral. Em pacientes com queimaduras maior que $20 \%$ da superfície corporal, a recomendação é de 0,3 a $0,5 \mathrm{~g} / \mathrm{kg}$ de peso por dia e deve ser administrada de 10 a 15 dias assim que a terapia for iniciada (Consenso forte - $95 \%$ de acordo). No trauma grave, a recomendação é de 0,2 a $0,3 \mathrm{~g} /$ $\mathrm{kg}$ de peso por dia podendo ser administrada nos primeiros 5 dias de nutrição enteral (Consenso forte - $91 \%$ de acordo). ${ }^{33}$

\section{Desempenho Físico e na Imunomodulação}

A administração oral crônica de L-glutamina livre ou do dipeptídeo L-alanyl-L-glutamina pode atenuar a lesão e a inflamação ocasionadas por exercícios exaustivos intensos e/ou aeróbicos.34 Além disso, administrações de aminoácidos de cadeia ramificada e/ou glutamina pode induzir elevação na concentração de fator de crescimento endotelial vascular e inibição do aumento de citocinas pró-inflamatórias após exercício agudo em atletas adolescentes. ${ }^{35}$

Recentemente, foi visto que a administração de glutamina tanto in vitro quanto in vivo atenuou os níveis pró-inflamatórios de genes e proteínas nos adipócitos e infiltração em tecido adiposo branco e macrófagos presentes nesse tecido. ${ }^{36}$ A glutamina também desempenha papel regulador e promove a polarização dos macrófagos M2, beneficiando a estabilidade das placas ateroscleróticas e inibindo a progressão das lesões. ${ }^{37}$

\section{Sobre Considerações Éticas - Decisões da Equipe Médica - Profissional da Saúde e de seus Pacientes}

Avanços científicos e tecnológicos têm sido cada vez mais pesquisados para a melhor qualidade de vida e saúde das pessoas, respeitando sua relação com a sociedade e com o meio ambiente. Fazer o bem e querer o bem deve ser uma meta saudável na relação do profissional de saúde e o paciente. O simples consentimento informado ou o termo de consentimento informado deve ser avaliado na relação durante a consulta/atendimento. A equipe profissional deve informar e esclarecer ao paciente cada intenção e procedimento, tranquilizando e envolvendo no contexto o paciente sob seus cuidados.

Naturalmente, o normal e o patológico devem ser considerados com referência às 
questões sócio-culturais e econômicas e mesmo cronológicas, levando-se em conta adicionalmente a prorrogação cada vez maior da fase do envelhecimento no ciclo de vida do ser humano.

Ademais, em diversas abordagens, existe uma previsão ou expectativa de benefício direto ou indireto e ainda o risco potencial, em grau variável, que precisa ser analisado no dia a dia do atendimento, junto ao paciente. Por outro lado, também, na atualidade, muito se discute sobre as subjetividades na ampla heterogeneidade das características físicas e metabólicas, fisiológicas e psicossociais e culturais dos indivíduos em uma população.

Existem padrões de normalidades fixas e bem estabelecidas no âmbito das áreas biomédicas, entretanto, deve-se alertar para alguns parâmetros e condições, nos quais identifica-se a falta de um limite claro entre as variações individuais de normalidade durante as diferentes fases do ciclo de vida e os pontos que determinam ou limitam os processos patológicos de um indivíduo sob cuidados por equipe de atenção à saúde.

\section{Uma Abordagem Criteriosa da Nutrologia e da Suplementação Nutricional}

Em uma abordagem translacional, dados preliminares de um trabalho de conclusão de curso da Universidade Federal da Bahia (UFBA), 38 de suplementação in vitro de glutamina em células de sangue periférico de indivíduos saudáveis, observou-se menor expressão de marcadores de superfície celular de linfócitos $\mathrm{T}$ e B nas 24 horas e maior expressão de CD45, marcador leucocitário após 18 horas. Os dados de uma tese doutoral mostraram uma modulação da resposta imune específica e ativação celular em leucócitos periféricos sob suplementação com L-GLN in vitro. ${ }^{39}$ Finalmente, dados preliminares, no modelo humano com estudo in vivo que resultaram de uma dissertação de mestrado no Programa de Pós-Graduação em Imunologia da UFBA, mostraram que após 10 dias de uso de GLN oral, indivíduos saudáveis jovens não mostraram alterações nos marcadores hepáticos, indicando função hepática preservada, níveis séricos mais altos de globulina e uma tendência de modular parâmetros imunológicos plasmáticos de citocinas de perfil de resposta celular tipo Th1.40

O Serviço de Nutrição Enteral e Parenteral (SENEP) do Hospital Santa Izabel nos últimos anos vem prescrevendo para pacientes com indicação de uso da L-GLN, com sucesso comprovado nos prognósticos de ganho de peso e de massa corporal, melhora de sinais clínicos analisados no acompanhamento da evolução clínica pré-e pós-cirúrgica, assim como no ambulatório de nutrologia, seguindo recomendações das associações internacionais e da SBNPE.

\section{Objetivo}

Neste trabalho de atualização de tema, os autores buscam elencar os principais achados da literatura nacional e internacional e fundamentar a importância básica e clínica para a leitura crítica da comunidade interessada no uso da glutamina.

\section{Material e Métodos}

Foi realizada uma análise crítica a partir de todos os artigos e referências utilizadas nos últimos anos na escrita de trabalhos de conclusão de curso, relatórios de iniciação científica, resumos de congressos e dissertações de mestrado e de tese doutoral, entre os trabalhos da linha de imunonutrição liderados pelos pesquisadores do grupo. Refeita a leitura de artigos selecionados no período 1996 até 2020. Todas as referências utilizadas seguiram recomendações e foram encontradas a partir do uso dos descritores em saúde (DeCS), do sistema de metadados médicos em língua inglesa (Medical Subject Headings - MeSH), a partir das palavras chave e termos cruzados em português e traduzidos ao inglês: L-glutamina, GLN, Q, imunonutrição, 
imunomodulação, hipertrofia, desnutrição, exercício físico, catabolismo, suplementação com GLN. Foram usadas a Biblioteca Virtual em Saúde (BVS) e as bases de dados da Biblioteca Nacional de Medicina dos Estados Unidos (NLM), o MEDLINE, Scientific Electronic Library Online (SciELO), Literatura Latino-Americana e do Caribe em Ciências da Saúde (Lilacs). Durante o levantamento dos trabalhos e pesquisas de novos artigos, encontramos 105 artigos, dos quais 9 entre os 79 foram selecionados por estarem diretamente associados com o uso da glutamina no modelo humano em estudo experimental in vivo.

\section{Resultados}

Dos artigos selecionados entre os encontrados com as características e foco de interesse, foram listados 9 artigos considerados no período de 2006 a 2019 (Quadro 1), com abordagem experimental in vivo, de administração/suplementação nutricional com GLN, apresentando as principais informações registradas pelos autores. Consideramos interessante ressaltar que os ensaios clínicos, ensaios clínicos duplo-cegos incluíam mais de um centro ou apresentavam dados com participantes em amostragem menor. Foram identificados 2 artigos de referência, com abordagem de experimental in vitro, com administração/suplementação de GLN em amostras de seres humanos.

Entre os artigos publicados no modelo in vivo, os autores trabalharam com participantes de seu convívio ou de sua área geográfica de atuação, tendo sido encontrados grupos de pesquisadores no Brasil (São Paulo, Brasília), Irã, Polônia, Reino Unido, Holanda, Coreia e grande grupo de clínicas de radioterapia oncológica (RTOG) associada ao Instituto Nacional de Câncer com clínicas em vários estados dos Estados Unidos.

O uso de suplementação de glutamina foi testado em condições de câncer, infecção, exercício físico de alta performance ou moderada, com grupos de atividades diferentes em cada artigo (Quadro 1).

\section{Discussão}

As informações trazidas na literatura analisada ilustram os mecanismos e principais achados sobre os aspectos biológicos da glutamina e aspectos funcionais diferentes em seus vários usos e aplicações.

A grande maioria dos autores expressa em suas conclusões o uso benéfico da glutamina nas diversas pesquisas encontradas a despeito do relatado por alguns pesquisadores que não encontraram os mesmos resultados relatados na literatura. Entretanto, esses últimos autores não apontam efeitos deletérios sobre o organismo, quando do uso da glutamina. Apenas encontram os efeitos favoráveis propostos nos objetivos iniciais do projeto.

O uso e o sucesso para uso da glutamina sob cada abordagem e demanda de suplementação com os dados encontrados foram majoritários.

Os resultados gerais de Alijani e Hosseini ${ }^{41}$ mostraram que a suplementação de glutamina pode ter um efeito positivo no sistema imunológico dos atletas durante o treinamento intensivo. Já Klek e colegas ${ }^{42}$ discutiram sobre a falha e as discrepâncias do aspecto nutritivo, uma vez que existem poucos ensaios clínicos focados na desnutrição em pacientes bem nutridos e seus resultados são contraditórios. Os benefícios da imunonutrição enteral perioperatória nesse estudo, em termos de redução de infecções pós-operatórias e menor tempo de internação hospitalar, foram semelhantes em pacientes bem nutridos e desnutridos (perda de peso de $10 \%$ ). Diferenças entre as formulações utilizadas nesse estudo e as adotadas por outros autores também podem ser responsáveis pelas discrepâncias observadas.

A imunonutrição parenteral demonstra uma variabilidade ainda maior, porque, na maioria dos casos, os imunonutrientes são introduzidos separadamente em formulações comerciais padrão, dependendo das necessidades individuais. $\mathrm{O}$ momento da intervenção nutricional é outra questão crítica que ainda 
precisa ser determinada. Segundo esses autores, mesmo que o presente estudo não tenha encontrado associação benéfica do uso rotineiro no pós-operatório de dietas imunoestimulantes em pacientes bem nutridos, um dos achados importante foi o fato de que a nutrição parenteral contemporânea pode ser administrada sem uma taxa de complicações mais alta em comparação com a via enteral. Considerando os custos mais altos e o aumento do uso de recursos devido à nutrição parenteral total, a terapia enteral deve ser considerada como o tratamento de escolha em todos os pacientes que necessitam de terapia nutricional.

Caris e $\operatorname{colegas}^{43}$ afirmam a alteração no balanço do perfil de resposta Th1 antes e após o exercício a uma altitude simulada de $4.500 \mathrm{~m}$. Neste sentido, a não modulação de basófilo e eosinófilos reforça a teoria de que existe uma tendência de induzir um perfil de resposta Th1 após a suplementação com GLN.

Berk e $\operatorname{colegas}^{44}$ não obtiveram dados conclusivos associando positivamente a glutamina à suplementação. A justificativa dos autores relacionou-se à perda de pacientes ao longo do ensaio clínico e perdas de peso dos pacientes não relacionadas à caquexia. $O$ estudo finalizou com $37 \%$ das 472 pessoas iniciais. Este estudo não foi capaz de testar adequadamente a capacidade de $\beta$-hidroxi $\beta$-metilbutirato, glutamina e arginina em reverter ou impedir a perda de massa magra entre pacientes com câncer. Os possíveis fatores contribuintes além da eficácia da intervenção foram: a) a incapacidade dos pacientes de concluir um curso de 8 semanas de tratamento e retornar em tempo hábil para avaliação de acompanhamento e b) os pacientes podem ter tido apenas perda de peso possível não relacionado à caquexia, mas a outras causas, como diminuição do apetite.

Para Bishay e colegas ${ }^{45}$ não houve diferença significativa entre os dois grupos no desfecho primário; evidência de invasão microbiana após 5 dias foi encontrada em 9/31 (grupo controle) e $8 / 29$ (grupo glutamina) (odds ratio 0,83
[0,24-2,86; $p=0,77])$.Sessenta bebês foram randomizados e atingiram o ponto final primário. Vinte e cinco pacientes apresentavam obstrução intestinal, 19 apresentavam defeitos na parede abdominal e 13 apresentavam enterocolite necrosante. Trinta e seis crianças apresentaram evidências de invasão microbiana durante $o$ estudo e 17 delas não foram detectadas pela hemocultura convencional. Mais da metade dos bebês cirúrgicos que necessitaram de NP apresentaram evidências de invasão microbiana. Aproximadamente metade disso não foi detectável pelas hemoculturas convencionais.

Finalmente, entre as pesquisas feitas sobre o uso da glutamina em suplementação no modelo humano, referente aos achados das ciências básicas, Spittler e colegas $^{46}$ associam que os baixos níveis de GLN desregulam a expressão de molécula de adesão (ICAM-1 / CD541, receptor Fc para IgGE. Nas amostras com glutamina, Coeffier e colegas ${ }^{18}$ apresentaram o papel deste aminoácido na imunidade inata, nos processos inflamatórios e na defesa imunológica. Assim, a modulação de processos inflamatórios tem sido também elencada entre as consequências do uso da glutamina. A heterogeneidade de resposta nas diferentes abordagens ou mesmo divergências entre opiniões médicas, clínicas e terapêutico-científicas deve-se a variação entre protocolos, abordagens metodológicas, marcas de produtos e diferença entre cada indivíduo, população, condição e momento da morbidade e saúde de base.

Os dados resgatados dos trabalhos analisados indicam maior positividade no uso pré-cirúrgico, pós-cirúrgico, em demanda da melhoria da condição física, na atividade muscular, na imunomodulação, tendo registrado a ativação de resposta de defesa com perfil Th1, ou seja, com ativação da imunidade celular. Pode ser importante contar com a experiência de análise e avaliação da evolução dos casos de cada grupo de pacientes para fins de incentivo de debates e discussão entre colegas das áreas afins e específicas multidisciplinares. 
Quadro 1. Dados extraídos dos artigos com abordagem metodológica in vivo e in vitro com foco na suplementação com GLN no modelo humano.

\begin{tabular}{|c|c|c|c|}
\hline $\begin{array}{l}\text { Informação sobre GLN - Principais } \\
\text { conclusões dos autores }\end{array}$ & Métodos & $\begin{array}{l}\text { Origem da população de estudo do artigo } \\
\text { E } \\
\text { Origem institucional dos autores }\end{array}$ & No. Ref \\
\hline \multicolumn{4}{|c|}{ Experimentos In vivo - administração/suplementação de GLN em seres humanos } \\
\hline $\begin{array}{l}\text { Os resultados não confirmam a ideia } \\
\text { de que o aumento da quantidade de } \\
\text { glutamina ou creatina poderia melhorar } \\
\text { a performance através da redução da } \\
\text { acidose metabólica muscular. Concluiu- } \\
\text { se que o treinamento resistido com } \\
\text { suplementação de creatina ou glutamina } \\
\text { não afetou a potência anaeróbia. }\end{array}$ & $\begin{array}{l}\text { Estudo experimental, duplo cego com abordagem } \\
\text { controlada por placebo para estabelecer comparação entre } \\
\text { grupos de voluntários suplementados com glutamina ou } \\
\text { com creatina e um grupo controle. Trinta e dois voluntários } \\
\text { sadios do sexo masculino com idade média de } 21,7 \pm 2,9 \\
\text { anos foram submetidos à avaliação antropométrica, de- } \\
\text { terminação da potência anaeróbia e avaliação nutricional } \\
\text { (PRÉ). Foram divididos, aleatoriamente, em três grupos. } \\
\text { Após oito semanas de treinamento com exercícios } \\
\text { resistidos com e sem suplementação, os voluntários foram } \\
\text { submetidos novamente às mesmas avaliações (PÓS), } \\
\text { conforme protocolo experimental esquematizado: } \\
\text { - GRUPO PLACEBO (PLA): Voluntários submetidos ao } \\
\text { programa de exercícios resistidos e ingestão de substância } \\
\text { placebo, } n=10 \text {. } \\
\text { - GRUPO GLUTAMINA (GLN): Voluntários submetidos ao } \\
\text { programa de exercícios resistidos suplementados com } \\
\text { glutamina, } n=11 \text {. } \\
\text { - GRUPO CREATINA (CRE): Voluntários submetidos ao } \\
\text { programa de exercícios resisti- dos suplementados com } \\
\text { creatina, } n=11 \text {. }\end{array}$ & $\begin{array}{l}\text { Trinta e dois voluntários sadios. } \\
\text { Trabalho realizado pelo autor na Faculdade de } \\
\text { Educação Física - Universidade de Brasília. }\end{array}$ & 48 \\
\hline 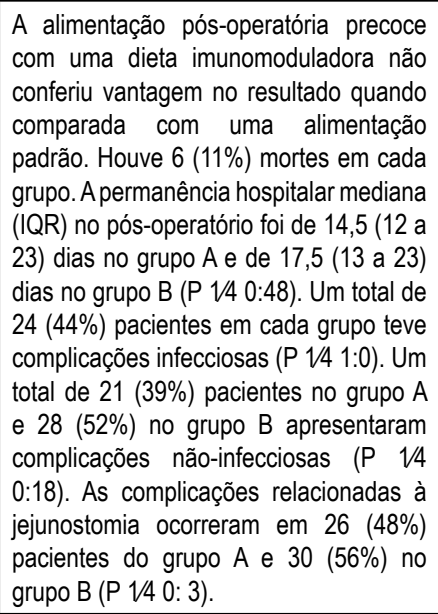 & $\begin{array}{l}\text { Estudo prospectivo, randomizado, controlado, duplo- } \\
\text { cego. Um total de } 120 \text { pacientes submetidos à ressecção } \\
\text { por câncer de pâncreas, esôfago e estômago foram } \\
\text { randomizados em duplo-cego para receber jejunostomia } \\
\text { com alimentação com dieta imunomoduladora (Stresson - } \\
\text { Grupo A) ou alimentos isonitrógenos e isocalóricos ( } 1250 \\
\text { calorias e } 75 \mathrm{~g} \text { de proteína / I - Nutrison High Protein - } \\
\text { Grupo B) por } 10 \text { a } 15 \text { dias. A alimentação foi iniciada } 4 \mathrm{~h} \\
\text { no pós-operatório e continuada por } 20 \mathrm{~h} / \text { dia. O volume } \\
\text { alvo ( } \mathrm{mL} / \mathrm{h} \text { ) foi de } 25 \text { no dia } 0,50 \text { no dia } 1 \text { e } 75 \text { a partir de } \\
\text { então. As medidas de desfecho incluíram complicações, } \\
\text { internação e mortalidade. }\end{array}$ & $\begin{array}{l}\text { Foram analisados } 108 \text { pacientes (54 em cada } \\
\text { grupo). Pacientes de três hospitais afiliados } \\
\text { à Faculdade de Medicina da Universidade de } \\
\text { Nottingham: Hospital Universitário, Nottingham, } \\
\text { Hospital da Cidade de Nottingham, Nottingham } \\
\text { e Derbyshire Royal Infirmary, Derby. } \\
\text { pacientes com idade entre } 30 \text { e } 80 \text { anos } \\
\text { submetidos à ressecção eletiva para câncer } \\
\text { gastrointestinal superior (esofágico, gástrico } \\
\text { e pancreático) entre janeiro de 2000 e junho } \\
\text { de } 2003 \text { foram incluídos no estudo. Section of } \\
\text { Surgery, University Hospital, Queen's Medical } \\
\text { Centre, E Floor, West Block, Nottingham NG7 } \\
\text { 2UH, UK Department of Surgery, Nottingham } \\
\text { City Hospital, Nottingham, UK Department of } \\
\text { Surgery, Derbyshire Royal Infirmary, Derby, UK } \\
\text { Trabalho realizado pelo grupo da Cirurgia de } \\
\text { Derby e Nottingham - no Reino Unido - }\end{array}$ & 47 \\
\hline $\begin{array}{l}\text { Os autores concluem que não } \\
\text { conseguiram provar a capacidade da } \\
\text { mistura de (HMB), ( } \beta \text {-hidroxil } \beta \text {-metil } \\
\text { butirato), glutamina earginina de prevenir } \\
\text { ou reverter a perda de massa magra do } \\
\text { indivíduo. Contudo, referem uma forte } \\
\text { tendencia a maior massa corporal em } \\
\text { pacientes usando o complexo }\end{array}$ & $\begin{array}{l}\text { Ensaio clínico de } 472 \text { pacientes com câncer avançado com } \\
\text { perda de peso entre } 2 \% \text { e } 10 \% \text { foram randomizados para } \\
\text { uma mistura de butirato de } \beta \text {-hidroxil } \beta \text {-metil, glutamina e } \\
\text { arginina ou uma mistura controle isocalórico isonitrógena, } \\
\text { tomada duas vezes por dia durante } 8 \text { semanas. } \\
\mathrm{HMB} / \mathrm{Arg} / \mathrm{Gln} \text { com } 7.72 \mathrm{~g} \mathrm{l-alanina,} 4.28 \mathrm{~g} \text { glicina, } \\
2.96 \mathrm{~g} \mathrm{l-serina,} 1.23 \mathrm{~g} \mathrm{l-ácido} \mathrm{glutâmico} \mathrm{e} 30.52 \mathrm{~g} \mathrm{de} \\
\text { gelatina. } \\
\text { Placebo administrado com solução isonitrogênea e } \\
\text { isocalórica. }\end{array}$ & $\begin{array}{l}\text { Pacientes que receberam tratamento em } \\
\text { qualquer membro integrante do grupo de } \\
\text { radioterapia oncológica Radiation Therapy } \\
\text { Oncology Group (RTOG). Instituto Nacional do } \\
\text { Câncer (NCl). } \\
\text { Centros de tratamento dos Estados Unidos - } \\
\text { Cleveland, Tampa, Miame, Bostom, Lebanon, } \\
\text { Arizona, Philadelphia. } \\
\text { Trabalho realizado pelo grupo das Instituições e } \\
\text { Centros do Grupo de radioterapia oncológica } \\
\text { Radiation Therapy Oncology Group (RTOG). } \\
\text { Instituto Nacional do Câncer (NCI) - Estados } \\
\text { Unidos. }\end{array}$ & 44 \\
\hline
\end{tabular}


Segundo os autores, com o estudo não se conseguiu demonstrar vantagem clara da imunonutrição pós-operatória de rotina em pacientes submetidos à cirurgia gastrointestinal superior eletiva.

As opções de tratamento enteral e parenteral mostraram eficácia, tolerância e efeitos similares na síntese de proteínas. A nutrição parenteral composta de acordo com as regras contemporâneas mostrou eficiência semelhante à nutrição enteral. No entanto, devido à sua relação custobenefício, a terapia enteral deve ser considerada como o tratamento de escolha em todos os pacientes que necessitam de terapia nutricional.

Os autores concluem sobre 0 efeito positivo de suplementação com GLN no treino intenso. Glutamina minimizando efeitos deletérios previstos e prováveis do treino/atividade física intensa .

Contrariamente, em recém-nascidos de baixo peso recebendo nutrição enteral suplementada com glutamina não houve aumento nas respostas linfocitárias Th1, como determinado in vitro, em avaliação subsequente, com células sanguíneas tratadas com aCD3/ aCD28 ou lipopolissacarídeo (LPS). De acordo com os autores, a menor razão de infecção em infantes sob suplementação de glutamina pode ser explicada por haver influências somente na mucosa, e não no sistema imunológico sistêmico.

Osautoresconcluemqueasuplementação de glutamina e carboidrato influencia o equilibrio Th1 / Th2 na resposta Th1 após 0 exercício a uma altitude simulada de $4.500 \mathrm{~m}$. E referem sobre estratégias nutricionais aumentaram a concentração sérica de IL-6, sugerindo um possivel efeito anti-inflamatório benéfico .

A suplementação parenteral mais a glutamina enteral não impede a invasão microbiana em bebês cirúrgicos. Contudo, a suplementação de glutamina ajuda na recuperação da imunoparesia pós-operatória e a glutamina impediu a invasão microbiana naqueles com a imunoparesia mais grave, sugerindo que uma população-alvo pode se beneficiar da suplementação no futuro.

\section{Estudo entre 2001 e 2005.}

Um grupo de 214 pacientes com gastrectomia ou pancreaticoduodenotomia, entre 18 e 80 anos, bem nutridos foi avaliado inicialmente para participar do estudo. Nove pacientes foram posteriormente excluídos e os 205 indivíduos restantes foram divididos aleatoriamente em esquema fatorial $2 \times 2 \mathrm{em} 4$ grupos de estudo: nutrição enteral padrão $(n=53)$, nutrição enteral imunomoduladora $(n=52)$, nutrição parenteral padrão $(n=49)$ e nutrição enteral imunomoduladora $(n=51)$.

Em modo duplo-cego (4 vezes, 45, 90, 135.180 minutos, cada 45 minutos $3,5 \mathrm{~g}$ todas as vezes). Grupo teste $14 \mathrm{gr}$. Glutamina. Grupo Placebo tomou maltodextrina. Grupos se exercitaram até a exaustão com um ergômetro e $75 \%$ do VO2 máx por 2 horas. 0 grupo controle não se exercitou.

Em um estudo duplo-cego, controlado por placebo, estudo controlado randomizado, lactentes com VLBW (very low birth weight) (idade gestacional $<32$ semanas e / ou peso ao nascer $<1500 \mathrm{~g}$ ) receberam suplementação enteral de glutamina $(0,3 \mathrm{~g} \mathrm{~kg} 1$ dia1) ou suplementação isonitrógena com placebo (alanina) entre os dias 3 e 30 de vida. As respostas de citocinas após estimulação in vitro das células sanguíneas totais com anti-(a) CD3 / aCD28 ou lipopolissacarídeo foram analisadas por agrupamento citométrico de esferas em 3 momentos: antes do início do estudo, no $7^{\circ}$ dia de vida e no $14^{\circ}$ dia de vida.

Nove homens foram submetidos a três sessões de exercício até exaustão com pico de VO2 a 70\% até a exaustão. Com sessões de hipóxia e suplementação durante os 6 dias antes do teste. Os homens consumiram $20 \mathrm{~g} / \mathrm{d}$ de probiótico de glutamina ou placebo $(10 \mathrm{~g}$ de amido de milho $+10 \mathrm{~g}$ de lactose) à noite.

Voluntários saudáveis e normais monitoração no setor e os exercícios foram realizados em camaras normobárica de simulação de altitude - Camara CAT - colorado altitude training CAT-12 - equivalente a pressão de $433 \mathrm{mmHg}$ e O2 13,5\%.

Foi utilizado um estudo prospectivo randomizado, duplocego, controlado, que estudou bebês cirúrgicos que receberam NP por pelo menos 5 dias com anomalias intestinais congênitas ou adquiridas (2009-2012).

Suplementação de GLN (parenteral mais enteral; total de $400 \mathrm{mg} / \mathrm{kg} / \mathrm{d}$ ) ou controle isonitrógeno. 0 desfecho primário foi a invasão microbiana avaliada após 5 dias de suplementação e definida como: (i) hemocultura convencional positiva, (ii) evidência de DNA microbiano no sangue (reação em cadeia da polimerase), (iii) nível de endotoxina plasmática e $50 \mathrm{pg} / \mathrm{mL}$ ou (iv) nível plasmático de proteína de ligação a lipopolissacarídeo e $50 \mathrm{ng} / \mathrm{mL}$. Os dados são apresentados em mediana (intervalo) e comparados por regressão logística.
Pacientes - grupo de 214 pacientes - (150 homens, 64 mulheres, idade média de 61,2 anos) com gastrectomia ou pancreaticoduodenotomia, entre 18 e 80 anos.

Trabalho realizado pelo grupo do $1^{\circ}$ Departamento de Cirurgia Geral Cracóvia, Polônia.

Grupo de 30 atletas, mulheres saudáveis em treino intenso de universidade do Irã.

Trabalho realizado pelo grupo da Faculdade de Educação Física - Chamran University - Irã.

Bebês com idade gestacional de 32 semanas ou menos ou peso igual ou inferior a $1.500 \mathrm{~g}$ que foram admitidos na unidade de terapia intensiva neonatal de nível III (UTIN) do VU University Medical Center, Amsterdã.

Trabalho realizado pelo grupo da Sofia Children, Health Institute Amsterdam, Rotterdam Holanda.

Voluntários saudáveis e normais.

Bebês com anomalias intestinais congênitas ou adquiridas.

Trabalho realizado pelo grupo colaborativo University College of London Great Ormond Street Institute of Child Health, London, United Kingdom; Great Ormond Street Hospital for Children, London, United Kingdom. Grupo de teste1 Cirurgia de glaucoma micro-invasiva (Trial Group1 Micro-Invasive Glaucoma Surgery - MIGS).

Trabalho realizado pelo grupo do Brasil Departamento de Fisiologia, Universidade Federal de São Paulo. 


\begin{tabular}{|l|l|}
\hline Neste estudo os autores sugeriram & Sete atletas adolescentes do sexo masculino realizaram \\
que as administrações de BCAA e / ou & $2.000 \mathrm{~m}$ de remo após a conclusão de cada três tratamentos \\
glutamina têm o suficiente para induzir & diferentes de 7 dias. O protocolo realizado com não \\
aumento da concentração de VEGF & tratamento (CO); uma dose diária total de aminoácidos \\
e inibir o aumento de citocinas pró- & de cadeia ramificada (Branched-chain amino acids BCAA) \\
inflamatórias após exercício em atletas & $3,15 \mathrm{~g}(1,05 \mathrm{~g} \times 3)$; ou uma dose diária total de BCAA 3,15 \\
da adolescência. & $\mathrm{g}(1,05 \mathrm{~g} \times 3)$ e glutamina $6 \mathrm{~g}(2 \mathrm{~g} \times 3)(\mathrm{BC}+\mathrm{GLU})$. Houve \\
& 30 dias período de descanso entre cada tratamento. Após \\
& a ingestão de aminoácidos por 7 dias, os participantes \\
& completaram o remo no dia 8, e suas amostras de sangue \\
& foram coletadas (em repouso, repouso; imediatamente \\
& após exercício Ex0; e 30 minutos após o exercício, Ex30) \\
& e foram analisados bioquimicamente usando ELISA.
\end{tabular}

Sete atletas masculinos adolescentes (idade: $17,7 \pm 0,74$ anos; altura: $175,3 \pm 1,38 \mathrm{~cm}$; peso: $67,5 \pm 4,87 \mathrm{~kg}$; IMC: $21,9 \pm 1,40 \mathrm{~kg} / \mathrm{m}^{2}$; gordura: $15,6 \pm 2,34 \%$; massa corporal magra: $56,3 \pm 2,54 \mathrm{~kg}$; carreira: 3,57 anos) participaram deste estudo.

Trabalho realizado pelo grupo dos laboratórios do Exercicio e Departamento de Educação Física em Busan, na Coreia. (Laboratory of Exercise Biochemistry, Department of Physical Education, Dong-A University, Busan, Korea Department of Rehabilitation Exercise \& Health, Busan Institute of Science and Techology).

Experimentos In vitro - administração/suplementação de GLN em amostras de seres humanos

A estimulação com IL-1b aumentou a IL-6 e a IL-8, mas não afetou a produção de IL-4 e IL-10. IL-8 e IL-6 produzidas de biópsias estimuladas diminuiu significativamente com 0 aumento da concentração de glutamina de 0,5 a $10 \mathrm{mM}$ para IL-6, ambos enquanto a produção de IL-10 foi aumentado de 0,7 para 1,2. A glutamina também aumentou o nível de mRNA da IL-10 em biópsias. Por outro lado, a produção de IL-4 não foi afetada pela glutamina. Conclusão: A glutamina foi demonstrada na mucosa intestinal humana para reduzir a produção de pró-inflamatórios citocinas IL-6 e IL-8 e aumentam a produção da citocina antiinflamatória IL-10.

A depleção de GLN foi associada a uma significativa redução do adenosina trifosfato celular (ATP), que pode ter influenciado a expressão do marcador da superfície celular e fagocitose. Reduzindo, baixando a concentração de GLN no meio de cultura de $2 \mathrm{mmol}$ a 200 $\mathrm{pmol} / \mathrm{L}$ reduziu a expressão de HLADR em $40 \%(P<0,001)$ em macrófagos derivados de monócitos e diminuição apresentação de antígeno induzido por toxoide tetânico.
Biópsias duodenais de oito indivíduos foram cultivadas com glutamina $0,5,2,5$ ou $10 \mathrm{mM}$ e estimulada com IL-1b $(2 \mathrm{ng} / \mathrm{mL})$. As placas de cultura foram colocadas em um câmara equilibrada com $5 \%$ de $\mathrm{CO}_{2}$ e incubada a $371 \mathrm{C}$ Após $18 \mathrm{~h}$, os meios de cultura foram preservados até 0 momento da análise. A produção de citocinas foi avaliada em meio de cultura por ELISA e expressão de mRNA de citocinas em biópsias por RT-PCR. Os resultados, em pg / $\mathrm{mg}$ de tecido, (mediana [intervalo]), foram comparados por testes pareados não paramétricos.

Os voluntários apresentaram um índice de massa corporal médio de $21,9 \mathrm{~kg} / \mathrm{m}^{2}$ (intervalo de 20,7 a 23,7 ). Todos os sujeitos estavam em bom estado geral saúde sem disfunção hepática, renal e cardíaca ou histórico médico digestivo ou cirúrgico. Biológico testes não revelaram sinais de estado inflamatório ou distúrbios metabólicos.

Separaçã de celulas e enriquecimento das PBMC e cultivo com diferentes cc de GLN
Voluntários saudáveis não fumantes (seis mulheres e seis homens; 19-39 anos) em estudo de endoscopia - normais. Os fragmentos de biópsias duodenais com peso médio de $8.171,9 \mathrm{mg}$ foram obtidas por endoscopia, após jejum noturno. Todos deram consentimento a participar do estudo aprovado pelo Comitê de Ética do Hospital Universitário de Rouen.

Trabalho realizado pelo grupo do Instituto de Aparelho Digestivo e Nutrição (ADEN EA 3234)

- Instituto das Pesquisas Multidisciplinares (IFR no23), CIC-INSERM-CHU Rouen, Cedex França.

População saudavel de Doadores de sangue (500mL) - Áustria - Viena.

Trabalho realizado pelo grupo do departamento de cirurgia e Instituto de Patologia experimentalDepartment of Surgery, Research Laboratories, and the Institute of General and Experimental Pathology, Universiy of Vienna,Austria.

Aparentemente, o conhecimento gerado com a pesquisa nas ciências básicas tem sido convergente com os achados clínicos. Entretanto, reforça-se a necessidade de aumento de pesquisas clínicas e translacionais.

\section{Conclusão}

Entre os trabalhos analisados disponíveis e encontrados sobre aspectos da análise da suplementação com GLN, o resultado geral na literatura aponta como favorável o seu uso. Nos artigos identificados, os autores trazem algumas variações nas abordagens de doses e tempos de uso nas diferentes morbidades ou demandas, embora essa variação encontrada tenha sido baixa e dentro do recomendado/preconizado internacionalmente. Não foram encontrados artigos com ênfase em aspectos negativos sobre o uso da glutamina, embora se deva considerar o cada caso com cuidado e acompanhamento clínico constante pela equipe médica que estabeleça o seu uso. 


\section{Agradecimentos}

Nossos agradecimentos às agências de bolsas de formação dos estudantes e estrutura organizacional dos setores administrativos e técnicos que possibilitam a realização de pesquisas nos setores do SENEP e do LABIMUNO da Universidade Federal da Bahia (UFBA).

\section{Referências}

1. Fürst P. New developments in glutamine delivery. The Journal of Nutrition. 2001 Sep 1;131(9):2562S-8S.

2. O'Neil, Maryadele J. The Merck index: an encyclopedia of chemicals, drugs, and biologicals. RSC Publishing, 2013.

3. Cruzat VF, Petry ÉR, Tirapegui J. Glutamina: aspectos bioquímicos, metabólicos, moleculares e suplementação. Revista Brasileira de medicina do Esporte. 2009 Oct;15(5):392-7.

4. Borges MC, Rogero MM, Tirapegui J. Enteral and parenteral supplementation with glutamine in preterm and low-birth-weight neonates. Revista Brasileira de Ciências Farmacêuticas. 2008 Jan 1;44(1):13-23.

5. TACO N. Tabela brasileira de composição de alimentos. Revista Ampliada NEPA. 2011.

6. Departamento de Informática em Saúde, Escola Paulista de Medicina (disponível emhttps://tabnut. dis.epm.br/alimento - acesso em 27 de fevereiro de 2020.

7. Cruzat VF, Rogero MM, Borges MC, Tirapegui J. Aspectos atuais sobre estresse oxidativo, exercícios físicos e suplementação. Revista Brasileira de Medicina do Esporte. 2007 Oct;13(5):336-42.

8. Newsholme P. Procopio J, Lima MM, Pithon-Curi TC, Curi R. Glutamine and glutamate - their central role in cell metabolism and function. Cell Biochem Funct. 2003;21:1-9.

9. van de Poll MC, Soeters PB, Deutz NE, Fearon KC, Dejong $\mathrm{CH}$. Renal metabolism of amino acids: Its role in interorgan amino acid exchange. The American Journal of Clinical Nutrition. 2004 Feb 1;79(2):18597.

10. D’Souza R, Powell-Tuck J. Glutamine supplements in the critically ill. Journal of the Royal Society of Medicine. 2004 Sep;97(9):425-7.

11. Rowbottom DG, Keast D, Morton AR. The emerging role of glutamine as an indicator of exercise stress and overtraining. Sports Medicine. 1996 Feb 1;21(2):8097
12. Labow BI, Souba WW, Abcouwer SF. Mechanisms governing the expression of the enzymes of glutamine metabolism-glutaminase and glutamine synthetase. The Journal of Nutrition. 2001 Sep 1;131(9):2467S$74 \mathrm{~S}$.

13. Pinel C, Coxam V, Mignon M, Taillandier D, Cubizolles C, Lebecque P, Darmaun D, Meynial-Denis D. Alterations in glutamine synthetase activity in rat skeletal muscle are associated with advanced age. Nutrition. 2006 Jul 1;22(7-8):778-85.

14. Roth E. Immune and cell modulation by amino acids. Clinical nutrition. 2007 Oct 1;26(5):535-44.

15.Lopes-Paulo-TSBCP FR. Efeitos da glutamina sobre a parede intestinal e sua aplicabilidade potencial em coloproctologia. Rev Bras Coloproct. 2005 Jan;25(1).

16. Xue H, Field CJ. New role of glutamate as an immunoregulator via glutamate receptors and transporters. Front Biosci (Schol Ed). 2011 Jun 1;3:1007-20.

17. Coqueiro AY, Rogero MM, Tirapegui J. Glutamine as an anti-fatigue amino acid in sports nutrition. Nutrients. 2019 Apr;11(4):863.

18. Coeffier M, Marion R, Ducrotte P, Dechelotte P. Modulating effect of glutamine on IL- $1 \beta$-induced cytokine production by human gut. Clinical Nutrition. 2003 Aug 1;22(4):407-13.

19. Horio Y, Osawa S, Takagaki K, Hishida A, Furuta T, Ikuma M. Glutamine supplementation increases Th1cytokine responses in murine intestinal intraepithelial lymphocytes. Cytokine. 2008 Oct 1;44(1):92-5.

20. Meynial-Denis D. Glutamine metabolism in advanced age. Nutrition reviews. 2016 Apr 1;74(4):225-36.

21. Cruzat V, Macedo Rogero M, Noel Keane K, Curi R, Newsholme P. Glutamine: metabolism and immune function, supplementation and clinical translation. Nutrients. 2018 Nov;10(11):1564.

22. De-Souza DA, Greene LJ. Intestinal permeability and systemic infections in critically ill patients: effect of glutamine. Critical care medicine. 2005 May 1;33(5):1125-35.

23. Sapidis N, Tziouvaras C, Ioannidis O, Kalaitsidou I, Botsios D. The effect of glutamine and synbiotics on the healing of colonic anastomosis. Rev Esp Enferm Dig. 2014 Apr 1;106(4):255-62.

24. Medina MA. Glutamine and cancer. The Journal of Nutrition. 2001 Sep 1;131(9):2539S-42S.

25. Waitzberg DL. Nutrição oral, enteral e parenteral na prática clínica v. 1. In Nutrição oral, enteral e parenteral na prática clínica v. 12009 (pp. 1289-1289).

26. Stonga ET, Bueno RZ, Naganota TA, Martins V, Rocha SL. Effects of intraperitoneal glutamine in the treatment of experimental sepsis. ABCD. Arquivos Brasileiros de Cirurgia Digestiva (São Paulo). 2019;32(2). 
27. Houdijk, A P J; van Leeuwen P A M,; Haarman HJThM (1998). Correspondence Authors' reply - Glutamineenriched enteral nutrition in patients with multiple trauma. Lancet.1998;352(7):1553.

28. Koeken VA, Lachmandas E, Riza A, Matzaraki V, Li Y, Kumar V, Oosting M, Joosten LA, Netea MG, van Crevel R. Role of Glutamine Metabolism in Host Defense Against Mycobacterium tuberculosis Infection. The Journal of Infectious Diseases. 2019 Apr 19;219(10):1662-70.

29. Rogero MM, Tirapegui J. Aspectos nutricionais sobre glutamina e atividade física. Nutrire. 2003;25:87112.

30. Foods And drugs Administration [homepage na internet]. FDA approved L-glutamine powder for the treatment of sickle cell disease [acesso em 01 jan 2020]. Disponível em: https://www.fda.gov/ drugs/resources-information-approved-drugs/fdaapproved-l-glutamine-powder-treatment-sickle-celldisease.

31. Vanek VW, Matarese LE, Robinson M, Sacks GS, Young LS, Kochevar M. Novel Nutrient Task Force, Parenteral Glutamine Workgroup. American Society for Parenteral and Enteral Nutrition (ASPEN) Board of Directors. ASPEN position paper: parenteral nutrition glutamine supplementation. Nutr Clin Pract. 2011 Aug;26(4):479-94.

32. Nunes ALB, Koterba E, Alves VGF, Abrahão V, Correia MITD. Projetos Diretrizes Associação Médica Brasileira e Conselho Federal de Medicina: Terapia Nutricional no Paciente Grave. 2011.

33. Singer P, Blaser AR, Berger MM, Alhazzani W, Calder PC, Casaer MP, Hiesmayr M, Mayer K, Montejo JC, Pichard C, Preiser JC. ESPEN guideline on clinical nutrition in the intensive care unit. Clinical Nutrition. 2019 Feb 1;38(1):48-79.

34. Raizel R, Tirapegui J. Role of glutamine, as free or dipeptide form, on muscle recovery from resistance training: a review study. Nutrire. 2018 Dec $1 ; 43(1): 28$.

35. BaeJY,Koo GH, ParkSC, Shin KO. Effects ofbranchedchain amino acid and glutamine supplementation on angiogenic factors and pro-inflammatory cytokines after acute exercise in adolescence athletes. The Asian Journal of Kinesiology. 2019 Apr 30;21(2):51-8.

36. Petrus P, Lecoutre S, Dollet L, Wiel C, Sulen A, Gao $\mathrm{H}$, Tavira B, Laurencikiene J, Rooyackers O, Checa A, Douagi I. Glutamine links obesity to inflammation in human white adipose tissue. Cell Metabolism. 2020 Feb 4;31(2):375-90.

37. Chen J, Zhang S, Wu J, Wu S, Xu G, Wei D. Essential role of nonessential amino acid glutamine in atherosclerotic cardiovascular disease. DNA and Cell Biology. 2020 Jan 1;39(1):8-15.
38. Neves EG. Descrição da cinética da expressão de marcadores celulares de linfócitos $\mathrm{T}$ após suplementação in vitro com L-glutamina de sangue periférico de indivíduos saudáveis. Salvador. Trabalho de Conclusão de Curso (TCC) em Biotecnologia - Universidade Federal da Bahia, UFBA. 2016.

39. Santos RGD. Diagnóstico imunológico da tuberculose humana utilizando antígenos recombinantes de Corynebacterium pseudotuberculosis. Salvador. Doutorado no Programa de Pos-Graduação em Imunologia - Universidade Federal da Bahia, UFBA. 2015.

40. Santos SQ. Avaliação do efeito modulador da glutamina na imunidade celular de pacientes submetidos à cirurgia oncológica. Salvador. Mestrado no Programa de Pos-Graduação em Imunologia - Universidade Federal da Bahia, UFBA. 2019.

41. Alijani E, Hossini Z. The effect of glutamine supplementation on immune system in female athlete students of Shahid Chamran University. World Journal of Sport Science. 2009;2(3):178-183.

42. Klek S, Kulig J, Sierzega M, Szybinski P, Szczepanek K, KubiszA, Kowalczyk T, Gach T, Pach R, Szczepanik $\mathrm{AM}$. The impact of immunostimulating nutrition on infectious complications after upper gastrointestinal surgery: a prospective, randomized, clinical trial. Annals of Surgery. 2008 Aug 1;248(2):212-20.

43. Caris AV, Lira FS, de Mello MT, Oyama LM, dos Santos RV. Carbohydrate and glutamine supplementation modulates the Th1/Th2 balance after exercise performed at a simulated altitude of $4500 \mathrm{~m}$. Nutrition. 2014 Nov 1;30(11-12):1331-6.

44. Berk L, James J, Schwartz A, Hug E, Mahadevan A, Samuels M, Kachnic L. A randomized, doubleblind, placebo-controlled trial of a $\beta$-hydroxyl $\beta$-methyl butyrate, glutamine, and arginine mixture for the treatment of cancer cachexia (RTOG 0122). Supportive Care in Cancer. 2008 Oct 1;16(10):117988.

45. Bishay M, Simchowitz V, Harris K, Macdonald S, De Coppi P, Klein N, Eaton S, Pierro A, MIGS Trial Group. The Effect of Glutamine Supplementation on Microbial Invasion in Surgical Infants Requiring Parenteral Nutrition: Results of a Randomized Controlled Trial. Journal of Parenteral and Enteral Nutrition. 2020 Jan;44(1):80-91.

46. Van den Berg A, van Elburg RM, Vermeij L, van Zwol A, van den Brink GR, Twisk JW, Nieuwenhuis EE, Fetter WP. Cytokine responses in very low birth weight infants receiving glutamine-enriched enteral nutrition. Journal of Pediatric Gastroenterology and Nutrition. 2009 Jan 1;48(1):94-101. 
47. Lobo DN, Williams RN, Welch NT, Aloysius MM, Nunes QM, Padmanabhan J, Crowe JR, Iftikhar SY, Parsons SL, Neal KR, Allison SP. Early postoperative jejunostomy feeding with an immune modulating diet in patients undergoing resectional surgery for upper gastrointestinal cancer: a prospective, randomized, controlled, double-blind study. Clinical Nutrition. 2006 Oct 1;25(5):71626.
48. Fontana KE. Efeito do exercício resistido associado à suplementação de creatina ou glutamina na potência anaeróbica. Revista Brasileira de Ciência e Movimento. 2008 May 29;14(3):79-86.

49. Spittler A, Winkler S, Gotzinger P, Oehler R, Willheim M, Tempfer C, Weigel G, Fugger R, Boltz-Nitulescu $\mathrm{G}$, Roth E. Influence of glutamine on the phenotype and function of human monocytes. Blood. 1995;86(4) (August 15):1564-1569. 